\title{
ASSOCIATION BETWEEN CENTRAL RETINAL THICKNESS AND VISUAL OUTCOME POST INTRAVITREAL RANIBIZUMAB INJECTION LOADING DOSES IN EXUDATIVE AGE RELATED MACULAR DEGENERATION
}

Ferdian Ramadhan, Ima Yustiarini, Adi Dwi Prakosa, Sauli Ari Widjaja, Wimbo Dwi Sasono, Muhammad Firmansjah Department of Ophthalmology, Faculty of Medicine Universitas Airlangga/ dr. Soetomo General Hospital, Surabaya, Indonesia

\begin{abstract}
Introduction: Age Related Macular Degeneration (AMD) stills the leading cause of blindness in developing countries for age 50 and above, increasing along higher life expectations. Anti-VEGF is the mainstay management for exudative AMD and able to reduce the central retinal thickness (CRT) and to increase the visual outcome. The aim of this study is to correlate between CRT and visual outcome in exudative AMD post intravitreal ranibizumab injection loading doses in Soetomo general academic hospital, Surabaya.
\end{abstract}

Methods: This was a retrospective, cross sectional study. Fourty two eyes who met inclusion criterias were received monthly loading doses of $0.5 \mathrm{mg}$ Ranibizumab intravitreal injection for three consequtives months. Baseline visual acuity (BCVA) was measured with Snellen Chart converted to LogMAR, CRT was measured by SD-OCT. Parameters was measured before the first injection and after completed three months injections.

Result: Fourty two eyes were composed by $59.5 \%$ male and $40.5 \%$ female with higher incidence in 61-70 years old (47.6\%). There was significant difference between CRT and BCVA before and after Ranibizumab injection ( $p<0.001$ ), mean BCVA were 0.831 before and 0.624 after injection. Mean CRT were 346.9 and $254.2 \mu \mathrm{m}$ before and after injection respectively. We found no correlation between CRT and BCVA before and after injection of Ranibizumab loading doses ( $p=0.418$ \& $p=0.275$ )

Conclusion: There were no correlation statistically between CRT and BCVA before and after injection of loading doses Ranibizumab intravitreal in exudative AMD. Further studies may be needed to prove the etiology of this tendencies.

\footnotetext{
Keywords: exudative age related macular degeneration; ranibizumab; anti-VEGF.

Cite This Article: RAMADHAN, Ferdian et al. ASSOCIATION BETWEEN CENTRAL RETINAL THICKNESS AND VISUAL OUTCOME POST INTRAVITREAL RANIBIZUMAB INJECTION LOADING DOSES IN EXUDATIVE AGE RELATED MACULAR DEGENERATION. International Journal of Retina, [S.I.], v. 4, n. 2, p. 169, sep. 2021. ISSN 2614-8536. Available at: <https://www.ijretina.com/index.php/ijretina/article/view/169>. Date accessed: 15 sep. 2021. doi: https://doi.org/10.35479/ijretina.2021.vol004.iss002.169.
}

\footnotetext{
Correspondence to:

Muhammad Firmansjah,

dr. Soetomo General Hospital,

Surabaya, Indonesia;

firmansjah.spm@gmail.com
}

\section{INTRODUCTION}

Age Related Macular Degeneration (AMD) is one of the leading causes of blindness in old age in developing countries for people over 50 years old. Along with the increase in life expectancy in old age, the incidence of AMD is increasing in countries around the world, especially developing countries. In general, AMD is divided into dry AMD and wet AMD where choroidal neovascularization (CNV) is the pathognomonic sign that differentiates between wet and dry AMD. Neovascularization will induce vascular leakage and bleeding, which can seriously affect the quality of the patient's vision. ${ }^{1,2,3}$ 
Several diagnostic modalities can be used for the detection of CNV in AMD. Optical Coherence Tomography (OCT) is a noninvasive modality that is able to diagnose, monitor and evaluate the progress of AMD. Compared to fluorescein angiography (FA), OCT has advantages in terms of scan speed, non-invasiveness and faster acute phase leak detection capabilities. Parameters that can be observed on OCT examination in AMD cases are central retinal thickness and the presence of fluid accumulation in the retinal layer.4,5

Vascular Endothelial Growth Factor (VEGF) is suspected as the main factor that causes the development of CNV so that inhibition of VEGF is an effective strategy for handling this case. Ranibizumab is a fragmentation of recombinant monoclonal antibody capable of neutralizing all active isoforms of VEGF-A which has been shown to be effective in increasing visual acuity in AMD patients and reducing central macular thickness in patients with AMD.6,7,8,9

In this study, a comparative study of visual acuity based on best corrected visual acuity (BCVA) using the LogMAR chart was conducted in patients with wet AMD and central retinal thickness (CRT) quantitatively using Spectral Domain OCT (SD-OCT) which was retrospectively assessed before and after Ranibizumab injection. $0.5 \mathrm{mg}$ intravitreal loading dose for 1 consecutive month in 3 months in Soetomo general academic hospital, Surabaya.

\section{METHOD}

This study is an analytic study with a cross sectional research design by taking secondary data from medical records retrospectively. We included the patient previously diagnosed with wet $A M D$ in either the right, left or bilateral eyes over 50 years old with complete examination data related to OCT and best corrected visual acuity at the time of examination. The patient had underwent three intravitreal loading doses of Ranibizumab at 1 month intervals consecutively for 3 months.
The exclusion criteria was patient with other diagnose of macular disorders, AMD with macular scar, wet AMD patients with other retinal disorders and patients who lost to follow-up thus underwent repeated intravitreal loading doses injections.

Injections were carried out by several vitreoretinal specialists at the same institution or ophthalmic residents under the supervision of a vitreoretinal specialist, injections were performed using a $30 \mathrm{G}$ needle and 1 cc syringe with sterile technique, $3.5 \mathrm{~mm}$ from the limbus edge for pseudophakic patients and $4 \mathrm{~mm}$ in phakic patients. Injection is done in the inferotemporal or superotemporal quadrant of the limbus.

We used Zeiss CirrusTM HD-OCT 5000 for analysis of central retinal thickness, average retinal thickness for the $500-\mu \mathrm{m}$-diameter circle, centered at the identified foveal location, was computed resorting to thin plate spline interpolation as identified from the respective colour fundus photograph. The OCT operation and interpretation before and after 3 consecutive months injections done by the same vitreoretinal specialist. BCVA in each eye was assessed according to the Snellen Chart and converted to the LogMAR Chart using the Snellen Equivalent assessed by eye residents under the supervision of refractive ophthalmologists.

Descriptive data from research results were collected in data collection sheets and then grouped, and presented in tabulation and diagrams using the mean \pm standard deviation. Data were collected and then assessed for distribution, to determine the normal distribution of data, the One Sample Shapiro-Wilk test was used. The research results are presented in the form of mean \pm standard deviation. To compare the best visual acuity with central retinal thickness before and after injection of intravitreal loading doses of Ranibizumab, the Wilcoxon test was used. To determine the best correlation between visual acuity and central retinal thickness before and 
after injection of loading doses of Ranibizumab, the Spearman test was used. The data is considered significant if the $p<0.05$. All statistical data was processed using SPSS 26.0 software. Ethical clearance approved by ethical committee of Soetomo general academic hospital.

\section{RESULT}

Based on the results of data collection, it was found that the number of samples was 42 eyes with a comparison of 25 samples of men $(59.5 \%)$ and 17 samples of women (40.5\%).Based on age category $50-60$ years as many as 7 samples (16.7\%), $61-70$ years as many as 20 samples (47.6\%), 71-80 years as many as 14 samples (33.3\%), 81 -90 years as much as 1 sample (2.4\%). There was no sample with exudative AMD in both eyes, 28 samples of the right eye $(66.7 \%), 14$ samples of the left eye $(47.6 \%)$ were found.

Table 1. Patient Characteristic

\begin{tabular}{cccc}
\hline Characteristics & Frequency & $\begin{array}{c}\text { Percentage } \\
(\%)\end{array}$ & $p$ \\
\hline Sex & 25 & 59.5 & 0.003 \\
Male & 17 & 40.5 & \\
Female & & & \\
Age & 7 & 16.7 & \\
$50-60$ & 20 & 47.6 & 0.001 \\
$61-70$ & 14 & 33.3 & \\
$71-80$ & 1 & 2.4 & \\
$81-90$ & 7 & 16.7 & \\
$50-60$ & & & \\
Laterality & 28 & 66.7 & \\
Right Eye & 14 & 47.6 & \\
Left Eye & 0 & 33.3 & \\
Bilateral & &
\end{tabular}

Pre-injection BCVA based on LogMAR value was 0.2 for 1 sample (2.4\%) and the best postinjection BCVA was 0.1 for 4 samples (9.5\%). The worst post-injection BCVA was 1.5 for 1 sample (2.4\%) and the worst post-injection BCVA was 1.5 for 3 samples (2.4\%). The mean
BCVA found was $0.831 \pm 0.33$ pre-injection and $0.624 \pm 0.41$ post-injection $(P=0.001)$ showed significant reduction of BCVA value post Ranibizumab serial injections. 
Table 2. Best Corrected Visual Acuity Pre and Post Ranibizumab Intravitreal Injection Loadaing Doses in Exudative AMD

\begin{tabular}{cccccc}
\hline & $\begin{array}{c}\text { Pre } \\
\text { Ranibizumab } \\
\text { Injection }\end{array}$ & $\begin{array}{c}\text { Percentage } \\
(\%)\end{array}$ & $\begin{array}{c}\text { Post } \\
\text { Ranibizumab } \\
\text { Injection }\end{array}$ & $\begin{array}{c}\text { Percentage } \\
(\%)\end{array}$ & $p$ \\
\hline BCVA & 0 & 0 & 4 & 9.5 & 0.001 \\
0.1 & 1 & 2.4 & 3 & 7.1 & \\
0.2 & 4 & 9.5 & 5 & 11.9 & \\
0.3 & 2 & 4.8 & 1 & 2.4 & \\
0.4 & 1 & 2.4 & 14 & 33.3 & \\
0.5 & 10 & 23.8 & 3 & 7.1 & \\
0.7 & 6 & 14.3 & 1 & 2.4 & \\
0.8 & 6 & 14.3 & 1 & 2.4 & \\
0.9 & 2 & 4.8 & 4 & 0 & \\
1.0 & 1 & 2.4 & 0 & 0 & \\
1.1 & 2 & 4.8 & 0 & 2.8 & \\
1.2 & 4 & 9.5 & 2 & 7.4 & \\
1.3 & 2 & 4.8 & 1 & 100 & \\
1.4 & 1 & 2.4 & 3 & \\
1.5 & 42 & 100 & 42 & $0.62 \pm 0.41$ & \\
Total & $0.83 \pm 0.33$ & \multicolumn{5}{c}{} \\
\hline Mean & 0 & &
\end{tabular}

Central retinal thickness from the sample results obtained pre-injection Ranibizumab was highest at 251-300 $\mu \mathrm{m}$ as many as 17 samples (40.5\%) and none below $200 \mu \mathrm{m}(0 \%)$. While the central retinal thickness after Ranibizumab injection showed that the most central retinal thickness was at 200-250 $\mu \mathrm{m}$, respectively 17 samples (40.5\%) and no samples with a thickness of 350- $400 \mu \mathrm{m}$ were found. The mean CRT for pre-injection of Ranibizumab was at $346.9 \pm 150.27 \mu \mathrm{m}$ and post-injection of Ranibizumab was at $254.2 \pm 110.9 \mu \mathrm{m}(\mathrm{p}<0.001)$ showed significant reduction of CRT after injection.

Table 3. Central Retinal Thickness Pre and Post Ranibizumab Intravitreal Injection Loading Doses in Exudative AMD

\begin{tabular}{cccccc}
\hline & $\begin{array}{c}\text { Pre } \\
\text { Ranibizumab } \\
\text { Injection }\end{array}$ & $\begin{array}{c}\text { Percentage } \\
(\%)\end{array}$ & $\begin{array}{c}\text { Post } \\
\text { Ranibizumab } \\
\text { Injection }\end{array}$ & $\begin{array}{c}\text { Percentage } \\
(\%)\end{array}$ & $p$ \\
\hline CRT & 0 & 0 & 10 & 23.8 & $<0.001$ \\
$<200 \mu \mathrm{m}$ & 6 & 14.3 & 17 & 40.5 & \\
$200-250 \mu \mathrm{m}$ & 17 & 40.5 & 10 & 23.8 & \\
$251-300 \mu \mathrm{m}$ & 4 & 9.5 & 2 & 4.8 & \\
$300-350 \mu \mathrm{m}$ & 9 & 21.4 & 0 & 7.1 & \\
$350-400 \mu \mathrm{m}$ & 6 & 14.3 & 3 & 100 & \\
$>400 \mu \mathrm{m}$ & 42 & 100 & 42 & $254.2 \pm 110.9$ & \\
Total & $346.9 \pm 150.27$ & \multicolumn{5}{c}{} \\
\hline Mean & \multicolumn{6}{c}{}
\end{tabular}


A Wilcoxon comparative test was conducted to compare the CRT pre and post-injection of Ranibizumab showing 42 negative ranks with a decrease in CRT results in all post-injection samples with a statistical significance of $<0.001$. The Wilcoxon comparative test also performed to compare BCVA pre and post Ranibizumab injection showing 34 negative ranks with a Decimal LogMAR BCVA meaning decreasing which means an increase in the patient's visual acuity after Ranibizumab injection. 6 Positive ranks which indicate an increase in decimal from LogMAR which means a decrease in visual acuity and 2 ties are found which means that the sample has no change in visual acuity after injection. The significance of this test is $<0.001$, which means that the statistical results are significant.

Table 4. Comparison of Central Retinal Thickness, Best Corrected Visual Acuity Pre and Post Ranibizumab Intravitreal Injection Loading Doses in Exudative AMD

\begin{tabular}{lcc}
\hline \multicolumn{1}{c}{ Variables } & $\mathrm{n}$ & $\mathrm{p}$ \\
\hline CRT Pre \& Post Injection & & \\
Negative Ranks & 42 & $<0.001$ \\
Positive Ranks & 0 & \\
Ties & 0 & \\
BCVA Pre \& Post Injection & & \\
Negative Ranks & 34 & $<0.001$ \\
Positive Ranks & 6 & \\
Ties & $\underline{2}$ & \\
\hline
\end{tabular}

Based on the results of the normality test, the Spearman correlation test was used to assess the relationship between BCVA and Ranibizumab pre-injection CRT which showed a significance of 0.418 (>0.05), which means the data is not significantly significant. Relationship between BCVA and CRT after Ranibizumab injection which showed a significance of $0.275(>0.05)$, which means the data was not statistically significant, there was also no relationship between BCVA and CRT after Ranibizumab injection.
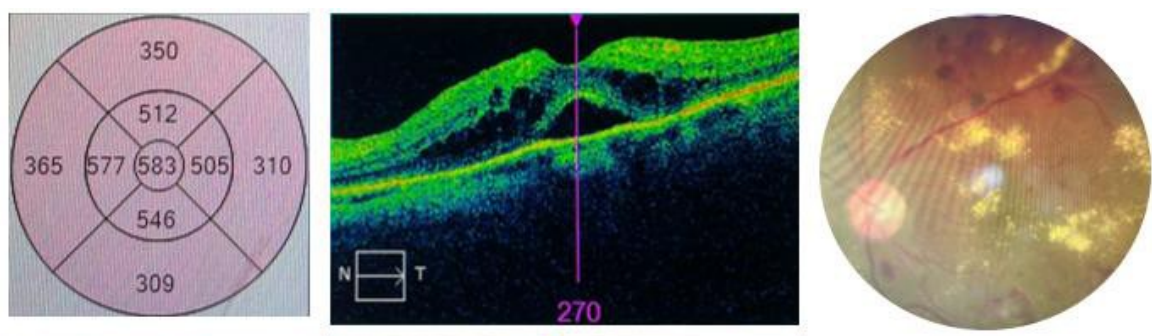

(A)
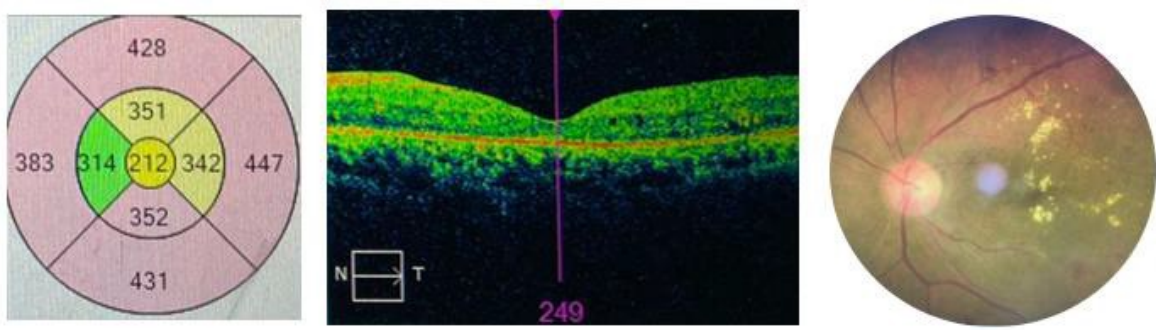

(B)

Figure 1. (A) Shows an increase in the thickness of the central retinal thickness with intra-retinal subretinal and sub-RPE fluid, the fundus photo shows the presence of exudate and macular edema (B) After three intravitreal ranibizumab injections shows a decrease in the central retinal thickness and the fundus photo shows a decrease of macular edema. In this patient, visual acuity changes only minimally, requiring RPE function testing to find the cause of minimal visual function changes 


\section{DISCUSSION}

According to research by Yonekawa and Kim, 2014 found from a data collection in the United States with the incidence of wet AMD as much as $0.4 \%$ at age $60-64$ years increasing to $8.2 \%$ over 80 years old. ${ }^{10}$ Another study from Kamao et al., 2020 found that from 165 samples, the mean age of the sample was at the age of $74.1 \pm 8.6$ with an age range of 50-95 years with a higher predilection for women than men. ${ }^{11}$ Study from Wong et al, 2020 which is a meta-analysis and global prevalence with 129,664 samples aged $30-97$ shows that the higher the age of the patient, the higher the incidence of exudative AMD. ${ }^{12}$ This is quite different from this study which relatively heterogen in each group of age, possibly because of the uneven distribution of the sample and the relatively small number of samples. The sample population in RSUD $d r$ Sutomo which is less heterogeneous with a stratified referral pattern could be one of the causes of the difference in the distribution of sample characteristics with other studies ${ }^{11,12}$

The mean BCVA found was 0.831 pre-injection and 0.624 post-injection. This shows that the visual acuity of the patients in the study was quite good and there was an increase in BCVA after intravitreal injection of Ranibizumab was performed. A study showed data from 236,843 eyes, and categorized them into six categories based on visual acuity. For visual acuity better than 20/80 (LogMAR 0.6), $77.59 \%$ of the samples were found which is directly proportional to this study, which showed that visual acuity in patients with exudative AMD was still relatively good. ${ }^{13}$ The MARINA and ANCHOR studies demonstrated that routine monthly injection of Ranibizumab increased mean best visual acuity (BCVA) by 20.5 and 21.5 letters, respectively, immediately after the third month of injection, as well as changes in OCT parameters in the HARBOR and PRONTO studies. ${ }^{14}$

Central retinal thickness results showed significant reduction after 3 consecutive months injections, compared to a study by Reznicek et al., 2014 showed that the average CRT before injection in patients with exudative AMD was around $278.07 \pm$ $87.56 \mathrm{~m}$ and significantly reduced from $71.22 \pm$ $106.93 \mu \mathrm{m}$ to $206.85 \pm 60.30 \mathrm{~m}$ after loading doses of anti-VEGF injection. ${ }^{15}$ Another study from De Almeida and de Souza, 2018 also showed a change from baseline $464.0 \pm 97.8 \mathrm{~m}$ to $365.50 \pm 95.1 \mathrm{~m}$ after 1 month of intravitreal Ranibizumab injection.

Association between BCVA and CRT preRanibizumab injection which showed a significance of $0.418(>0.05)$, which means the data was not statistically significant, there was no relationship between BCVA and CRT pre-Ranibizumab injection, as well as the relationship between BCVA and CRT after Ranibizumab injection which showed a significance of 0.275 . ( $>0.05)$ which means the data is not statistically significant. Our findings showed that OCT - CRT measurements, although able to quantify the height and volume of macular oedema, cannot be used alone as a reliable indicator of visual acuity loss. Thing that might potentially lead to this negative correlation is the retinal photoreceptor status that occurs over time with the progression of AMD or the time from initial diagnosis until the patient received the initial injection can triggered the progressive damage of retinal photoreceptor cells.

A study from Ou et al., 2017 which correlated visual acuity with central retinal thickness after antiVEGF administration in several retinal disorders showed a very minimal correlation between BCVA and CRT in AMD cases at baseline and no correlation was found between BCVA and CRT. at month 12 . Another study from Puell et al., 2020 also showed no association between CRT and best visual acuity in patients with AMD. One of the factors that causes this is the damage to the function of the RPE itself which results in damage to central visual acuity so that it is necessary to test the function of the RPE and other functional visual tests. 
Damage to the RPE function will result in other visual disturbances. It should be noted that mesopic or low illuminance, low luminance deficit (LLD) and the difference between photopic and mesopic visual acuity will be impaired in patients even from early AMD. It is necessary to do a visual acuity test compared to CRT under photopic and mesopic luminance conditions and then compared with normal conditions which with increasing age are also known to change physiologically in these functions.

Subsequent studies are slightly contradictory but some points are linear with the above comparison. The study of Danis et al., 2015 showed a negative correlation between CRT and changes in BCVA in cases of exudative AMD at day 90 and day 180 post anti-VEGF injection. Although changes in BCVA often coincide with increases in CRT, the relationship between the two is still not well understood. This study also stated that the correlation between the two was not linear for all cases of macular edema, the relationship between BCVA and CRT was also found when there was a decrease in CRT to $250 \mathrm{~m}$ or more indicating a complex relationship that should be investigated further.

A limitation of this study is the shorter follow-up time when compared to other studies to assess changes in BCVA and CRT which may be assessed up to 1-2 years. To make measurements in a longer time it is also possible to require a larger number of samples with more complete follow- up data. Selection bias is also a potential limitation of this study in terms of early diagnosis of AMD samples before the initial injection or in detecting other abnormalities in the retina, especially the macula. Author hoped that future research can dig up the informations and holistic examination of retinal function test to sharpen the causal relationship between CRT and visual acuity.

\section{CONCLUSION}

BCVA pre and post Ranibizumab intravitreal injection loading doses in exudative AMD of 3 consecutive months showed marked enhancement and CRT showed significant reduction but correlation of BCVA and CRT Pre and Post Ranibizumab injection were unsignificant. Further studies are needed to determine the causative factors in order to determine further visual prognostic and the decision to continue the injections.

\section{REFERENCES}

1. Riordan-Eva, P. 2018. Anatomy \& Embryology of the Eye. In: P. Riordan-Eva, J. J. Augsburger (Eds.). Vaughan \& Asburry's General Ophthalmology. 19th Ed. Mc-Graw Hill Education. New York. 32 - 38.

2. Brar, V. S., Law, S. K., Lindsey, J. L., Mackey, D. A., Schultze, R. L., Singh, R. S. J., Silverstein, E. 2019. The Eye. In: Fundamentals and Principles of Ophthalmology. Basic and Clinical Science Course 2019 - 2020. American Academy of Ophthalmology. San Fransisco. 119 - 138.

3. McCannel, C. A., Berrocal, A. M., Holder, G. E., Kim, S. J., Leonard, B. C., Rosen, R. B., Spaide, R. F., Sun, J. K. 2019. Basic Anatomy. In: Retina and Vitreous. Basic and Clinical Science Course 2019 - 2020. American Academy of Ophthalmology. San Fransisco. $31-47$.

4. Regatieri, C. V., L. Branchini, J.S. Duker. 2011. The role of spectral-domain OCT in the diagnosis and management of neovascular age-related macular degeneration. Ophthalmic Surg Lasers Imaging. 42. 1-18. doi: 10.3928/15428877- 20110627-05. 
5. Michalewski, J., J. Nawrocki, B. Izdebski, Z. Michalewska. 2014. Morphological changes in spectral domain optical coherence tomography guided bevacizumab injections in wet age-related macular degeneration, 12-months results. Ind J Ophth. 62(5), 554-560. doi: 10.4103/03014738.133485.

6. Sayed, K. M., T. Naito, T. Nagasawa, T. Katome, Y. Mitamura. 2011. Early visual impacts of optical coherence tomographic parameters in patients with age-related macular degeneration following the first versus repeated Ranibizumab injection. Graefes Arch Clin Exp Ophthalmol. 249(10)1449-1458. doi: 10.1007/s00417011-1672-2.

7. Kato, A., T. Yasukawa, Y. Ogura. 2014. Antivascular endothelial growth factor therapies for neovascular age-related macular degeneration: Search for the optimized treatment regimen.Taiw J Ophthalmol. 4(2014). 3-8. doi: 10.1016/j.tjo.2013.12.003

8. Sizmaz, S., C. Kucukerdonmez, A. Kal, E.Y. Pinarci, H. Canan, G. Yilmaz. 2014. Retinal and choroidal thickness changes after single anti-VEGF injection in neovascular age-related macular degeneration: Ranibizumab vs bevacizumab. Eur J Ophthalmol. 24(6). 904-910. doi: 10.5301/ejo.5000478.

9. McCannel, C. A., Berrocal, A. M., Holder, G. E., Kim, S. J., Leonard, B. C., Rosen, R. B., Spaide, R. F., Sun, J. K. 2019. Basic Anatomy. In: Retina and Vitreous. Basic and Clinical Science Course 2019 - 2020. American Academy of Ophthalmology. San Fransisco. $31-47$.

10. Yonekawa, Y. and Kim, I.K. 2015. Clinical Characteristics and Current Treatment of Age-Related Macular Degeneration. Cold
Spring Harb Perspect Med. doi: 10.1101/cshperspect.a017178

11. Kamao, H., K. Goto, K. Matsuno., K. Mizukawa, A. Miki, J. Kiryu. 2021. Clinical Characteristics of Neovascular Age-Related Macular Degeneration without Typical. Hindawi J. Ophthalmol. 2021,1-8. doi: $\underline{10.1155 / 2021 / 6683532}$

12. Wong, W. L., Su, X., Li, X., Cheung, C. M. G., Klein, R., Cheng, C.-Y., \& Wong, T. Y. 2014. Global prevalence of age-related macular degeneration and disease burden projection for 2020 and 2040: a systematic review and meta-analysis. The Lancet Global Health, 2(2), e106-e 116. doi:10.1016/s2214$109 \times(13) 70145-1$

13. Ho, A.C., Kleinman, D.M., Lum, F.C., Heier J.S., Lindstrom ,R.L., Orr, S.C., Chang G.C., Smith, E.L., Pollack J.S. 2020. Baseline Visual Acuity at Wet AMD Diagnosis Predicts Long-Term Vision Outcomes: An Analysis of the IRIS Registry. Ophthalmic Surg Lasers Imaging Retina. 2020(51):633-639. doi: 10.3928/23258160-20201104-05

14. Gale, R., Korobelnik, J.-F., Yang, Y., \& Wong, T. Y. 2016. Characteristics and Predictors of Early and Delayed Responders to Ranibizumab Treatment in Neovascular Age-Related Macular Degeneration: A Retrospective Analysis from the ANCHOR, MARINA, HARBOR, and CATT Trials. Ophthalmologica, 236(4), 193-200. doi:10.1159/000451065

15. Reznicek, L., Muhr, J., Ulbig, M., Kampik, A., Mayer, W. J., Haritoglou, C., ... Wolf, A. 2014. Visual acuity and central retinal thickness: fulfilment of retreatment criteria for recurrent neovascular AMD in routine clinical care. British Journal of Ophthalmology, 98(10), 1333-1337. doi:10.1136/bjophthalmol-2013-304399 
16. De Almeida, N. A. and O. F. de Souza. 2018. Early changes in macular optical coherence tomography parameters after Ranibizumab intravitreal injection in patients with exsudative age-related macular degeneration, Int J Retin Vitr. 4(1), 1-5. doi: 10.1186/s40942-018-0109-z.

17. Ou, W. C., Brown, D. M., Payne, J. F., \& Wykoff, C. C. 2017. Relationship Between Visual Acuity and Retinal Thickness During Anti-Vascular Endothelial Growth Factor Therapy for Retinal Diseases. American Journal of Ophthalmology, 180, 8-17. doi:10.1016/j.ajo.2017.05.014

18. Puell, M. C., Hurtado-Ceña, F. J., PérezCarrasco, M. J., \& Contreras, I. 2020. Association between central retinal thickness and low luminance visual acuity in early age-related macular degeneration. European Journal of Ophthalmology, 112067212096874. doi:10.1177/1120672120968740

19. Danis, R. P., Sadda, S., Jiao, J., Li, X.-Y., \& Whitcup, S. M. (2016). Relationship between retinal thickness and visual acuity in eyes with retinal vein occlusion treated with dexamethasone implant. Retina, 36(6), 1170- 1176. doi:10.1097/iae.0000000000000851

\section{@ $\odot \Theta \Theta$}

This work licensed under Creative Commons Attribution 